\title{
APUNTES TEÓRICO-METODOLÓGICOS PARA ABORDAR LA DUPLA JÓVENES-ESPACIO PÚBLICO
}

\author{
Theoretical-Methodological Notes to Address the Relationship between Youth and Public Space
}

\author{
Marcela Meneses-Reyes \\ Jahel López-Guerrero
}

\begin{abstract}
Resumen: El presente artículo tiene como objetivo exponer algunos apuntes teórico-metodológicos que se han identificado como necesarios para impulsar una línea de investigación centrada en construir un vínculo teórico y analítico acerca de la relación de las y los jóvenes con el espacio público, atendiendo tanto al campo de estudio sobre juventudes, como a los estudios sobre el espacio en las ciencias sociales, con el objetivo de construir conocimiento de mayor alcance analítico, que a su vez pueda comunicarse a los tomadores de decisiones para su posible traducción en política pública.
\end{abstract}

Palabras clave: jóvenes, espacio público.

Abstract: In this article, we discuss theoretical-methodological notes that we have identified as necessary in an effort to promote a line of research -in studies about both youth and space in the social sciences - focused on building a theoretical and analytical basis about how young people relate to public space. Our aim is to build more in-depth knowledge of greater analytical scope that, in turn, will be communicated to decision makers for possible translation into public policy.

Keywords: young people, public space.

Marcela Meneses Reyes. Doctora en Ciencias Políticas y Sociales con
orientación en Sociología por la Universidad Nacional Autónoma de
México. Profesora-investigadora en el Instituto de Investigaciones
Sociales de la Universidad Nacional Autónoma de México. Temas
de especialización: jóvenes, espacio público, conflictos urbanos,
acción colectiva y movimientos sociales. Correo electrónico: marcela.
meneses@sociales.unam.mx.
Jahel López Guerrero. Doctora en Antropología por la Universidad
Nacional Autónoma de México. Profesora-investigadora en el Centro de Investigaciones Interdisciplinarias en Ciencias y Humanidades de la Universidad Nacional Autónoma de México. Temas de especialización: mujeres indígenas jóvenes, ciudades seguras para las mujeres, mujeres en el ámbito de la seguridad pública. Correo electrónico: jahellg1973@ yahoo.com.mx.

Enviado a dictamen: 6 de diciembre de 2017

Aprobación: 17 de abril de 2018

Revisiones: 1 


\section{Introducción}

E propósito de este artículo es dar continuidad a una discusión teórica y metodológica para analizar la influencia que ejercen los actores juveniles en la producción del espacio público y, a su vez, la influencia que despliega la dimensión espacial en la configuración de distintas formas de ser y vivir la juventud.

Para ello, exponemos en la primera parte diversos hallazgos basados en un primer acercamiento a la literatura identificada acerca de dicha relación, para posteriormente desarrollar unas notas teóricometodológicas iniciales que apuntan a caracterizar al sujeto joven y a colocar la experiencia juvenil en el centro del análisis, esto como una tarea básica a realizar en una indagación que pretenda estudiar la vinculación de este sujeto con el espacio público. Proponemos estas notas a partir de la experiencia de las autoras como investigadoras de la condición juvenil en el espacio urbano de la Ciudad de México, pero también como resultado de la reflexión a propósito de dos seminarios organizados en los dos últimos años. ${ }^{1}$

Las ponencias presentadas en ambos seminarios conformaron un mosaico de investigaciones realizadas en torno a heterogéneas identidades y condiciones juveniles, así como en distintos espacios y territorios. Concretamente, en la frontera sur se analizaron fenómenos ocurridos en San Cristóbal de Las Casas, Tuxtla Gutiérrez, Tapachula y San Juan Chamula, en Chiapas; en la capital de Oaxaca y en el municipio de Tlahuitoltepec; y en las periferias de Cancún, Quintana Roo; asimismo, se abordaron estas mismas temáticas en otros estados como Nuevo León, San Luis Potosí, Baja California Norte y el Estado de México, y en ciudades de otros países como Bogotá, Granada y Barcelona. Los espacios analizados igualmente presentaban enorme diversidad en cuanto a las calles, el transporte público, el espacio transmigratorio, los espacios organizativos, el espacio escolar, la asamblea comunitaria, el escenario, el centro de la ciudad, el barrio y la ciudad misma. Por último, observamos en los análisis diferentes concatenaciones de la condición juvenil con el género, la orientación sexual, la etnia y la clase, lo cual dinamiza la posición y marca diferentes niveles de desigualdad en el acceso, uso, apropiación y disfrute de los jóvenes ${ }^{2}$ del espacio público.

\section{Aproximaciones a la dupla jóvenes-espacio público}

En el campo de estudio sobre jóvenes, así como en el estudio del espacio público, se ha establecido una relación que ha sido objeto de análisis, aunque como tal no se ha generado una línea de investigación a la que se adscriba una comunidad epistémica reconocida por centrar su atención en la dupla mencionada, la cual se explica más adelante. No obstante, un primer acercamiento a la literatura encontrada hasta el momento en Iberoamérica ha permitido distinguir diversos ángulos de aproximación a la relación entre los jóvenes y el espacio público, cuyo foco de atención se centra en lo espacial como contenedor de presencias y expresiones o como eje configurador de experiencias; $\mathrm{o}$ en los sujetos jóvenes con un papel activo en las maneras de usar, disputar y significar el espacio público urbano. A partir de lo anterior, hasta el momento, resaltan las siguientes cuatro áreas de problematización:

1. Jóvenes en el espacio público como resultado de sus condiciones de vulnerabilidad y exclusión. Los autores que han desarrollado esta línea reconocen que las condiciones estructurales en los países iberoamericanos resultan adversas para las mayorías, lo cual impacta en las experiencias de los jóvenes pertenecientes a los sectores populares cuya presencia en las calles es resultado y expresión de su situación de vulnerabilidad y exclusión (Saraví, 2004 y 2015; Eseverri, 2015). En esta línea podrían adscribirse desde los trabajos iniciales en el campo de los estudios sobre juventud de la década de los ochenta del siglo pasado, los cuales analizaron la presencia de las "bandas" en los barrios de las periferias urbanas como resultado de un sistema económico y político cada día más indiferente ante los jóvenes, hasta la expresión extrema de dicha problemática en los jóvenes en situación de calle 
(Makowski, 2007; Espinosa, 2009 y 2012; Pérez y Barragán, 2012). Además, existe una corriente que apela a la investigación-acción para intervenir en el espacio público con el fin de paliar las situaciones de exclusión en las que se encuentran los jóvenes con el fin de mejorar sus condiciones de vida (Omaña, 2011; Víquez y Rojas, 2006).

2. Jóvenes en el espacio público de la violencia, el miedo y la inseguridad. En este campo se expresa nítidamente la perspectiva de la cual se parte para analizar la relación de los jóvenes con el espacio público, ya sea desde una posición adultocéntrica ${ }^{3}$ que impone los tiempos, los horarios, los modos, las prácticas y el tipo de sujetos a los que les es permitido el acceso a ciertos espacios, relegando a todo aquel que rompa con dicha regulación ${ }^{4}$ (Pérez, Salcedo y Cáceres, 2012), o desde esta misma posición adultocéntrica que concibe a los jóvenes como sujetos amenazantes, riesgosos e indeseables y que, por lo tanto, les niega toda posibilidad de acceso, uso, decisión y disfrute (Jasso, 2015); o lo que describe Vommaro (2014) con relación a los rolezinhos en Sao Paulo, Brasil. En sentido inverso, opera una perspectiva que desde nuestro punto de vista refuerza una concepción sobre los jóvenes como víctimas (Meneses, 2008; Reguillo, 2012b; Valenzuela, 2015; Ravelo y Domínguez, 2010), y que se muestra aún más en cuanto a las problemáticas que padecen las mujeres jóvenes, principalmente relacionadas con la violencia sexual. Finalmente, está presente otra corriente que problematiza las tensiones, contradicciones y reproducciones que en las lógicas de la violencia ejercen los mismos jóvenes como sujetos activos, y en muchas ocasiones actuantes de la violencia al mismo tiempo (Rodríguez, Solares y Zabala, 2009).

3. Protesta, acción colectiva y participaciónjuvenilenelespacio público. En este campo aparece el espacio público en segundo término, como un contenedor de prácticas, demandas y acciones encabezadas por los jóvenes en búsqueda de ciertos objetivos, principalmente políticos. Aquí se inscriben los análisis sobre movimientos estudiantiles, y más recientemente sobre el \#YoSoyl32 en México, así como las movilizaciones estudiantiles en Chile de la llamada "generación sin miedo" (Berroeta y Sandoval, 2014; Urzúa, 2015). Asimismo, existe una corriente que apela a la concepción del espacio público como un espacio de lo común, en el cual aparecen los jóvenes como sujetos centrales que trascienden al Estado y que ponen en práctica nuevas formas de participación y acción política (Vommaro, 2014). Paralelamente se ha desarrollado una concepción de los jóvenes movilizados en el espacio público como parte de un proceso de construcción de ciudadanía, en donde el Estado aparece como un actor central de interpelación (López, 2017; Morfín, 2011; Zenil, 2010; Batallán et al., 2009). También se está analizando el papel de Internet, las nuevas tecnologías y las redes sociales en la vinculación de los jóvenes con el espacio público (Tully y Alfaraz, 2012), lo cual nos lleva a proponer una modalidad de acercamiento al espacio público desde el plano de lo virtual, recuperando el método de la etnografía virtual propuesto por Christine Hine (2000) y de la cibernografía propuesta por Rossana Reguillo (2012a). El papel de Internet y de las redes sociales en las nuevas formas de incidencia política de los jóvenes es una de las grandes interrogantes que se debaten actualmente. De este planteamiento se desprenden los análisis de Rossana Reguillo (2012c), Guiomar Rovira (2013a, 2013b y 2014), Benjamín Arditi (2015) y Portillo (2014), por mencionar algunos.

4. El espacio público como escenario para el ocio, el arte y la cultura juvenil. El impacto que tuvo el grafiti en el mundo, sobre todo en la década de los noventa, visibilizó las disputas permanentes en torno al espacio público que se dirimen entre una diversidad de actores entre los que resaltan los jóvenes, quienes entablan una relación dinámica, conflictiva y constantemente resignificada en torno a la ciudad y su manera de estar en ella, lo cual llevó a los gobiernos a la creación de reglas y normas prohibicionistas, ${ }^{5}$ por un lado, o, por otro lado, a la institucionalización de la práctica con 
fines de regulación, y hasta de autolegitimación, de los gobiernos en apariencia democráticos (Meneses y Franco, 2018). Sin embargo, los jóvenes siguen irrumpiendo creativamente en el espacio público, como lo han demostrado un sinfín de análisis sobre grafiti y arte callejero (Lopera y Coba, 2016; Meneses, 2016b). A su vez, se ha problematizado desde este ángulo a partir de distintas experiencias como la generación de espacios públicos autogestivos por parte de los propios jóvenes (Ortega, Lazcano y Teixeira, 2015), y la apropiación de espacios públicos con fines de diversión y entretenimiento, tales como los centros comerciales, centros culturales, tianguis y mercados, parques, cafés internet y plazas (Bohórquez, López y Suárez, 2016; Urteaga, 2013, Meneses, 2016a; López, 2017; Hernández, 2001; Castro y Zepeda, 2004), o con fines de control y ejercicio de prácticas violentas.

Estamos conscientes de que tanto en el campo de estudio sobre juventudes, como en el de los estudios sobre el espacio, se han hecho en el primer caso alusiones a la importancia que tiene para los jóvenes el espacio público urbano y, en el segundo, a las formas en que el espacio influye, ya sea posibilitando o restringiendo la experiencia de lo juvenil. De este modo, el reconocimiento de una mutua relación entre estas dos dimensiones muestra inicialmente dos diferentes caminos de abordaje que se presentan a continuación.

\section{Desde lo juvenil}

Desde este enfoque habría que revisar cómo se define este concepto: como una transición hacia la vida adulta, que de tal modo coloca a los jóvenes en una posición subordinada bajo el imperativo del "deber ser", o como una experiencia situada en el presente y que se expresa en una diversidad de formas de "ser" joven, con capacidad para reproducir, pero igualmente para producir cultura y vida social. En este caso las alusiones al espacio público mostrarían, para la primera perspectiva, las normas que debieran seguir o la prohibición del rompimiento de las mismas para participar en dicho espacio. En la segunda perspectiva podría pensarse en temáticas que ayuden a observar cómo los jóvenes se construyen como tales en el ámbito espacial, y cómo éste impone restricciones o posibilidades para llevar a cabo dicho proceso. La idea sería no decantarse por una u otra perspectiva, sino más bien observar las tensiones, disputas y convergencias entre el deber ser desde la mirada adulta y el estar siendo desde la perspectiva de los jóvenes. En este sentido, vale la pena replicar análisis como el de Mariana Chaves (2010), quien propone que el estudio de la experiencia de los jóvenes en el espacio público es central porque dispone al sujeto joven a desmarcarse del mundo privado e incorporarse al público, de suerte que habrá que analizar los procesos de socialización en espacios homogéneos, así como los circuitos de sociabilidad que construyen los jóvenes dentro, en los límites o fuera de estos espacios, porque en ellos se reproducen desigualdades sociales y se expanden los miedos en la ciudad, con incidencia en las familias, en formas de socialización y en formas de endoculturación particulares, que en conjunto propician la anulación de los contactos con lo diferente por desigualdad o diversidad; de esta manera, sostiene la autora, pueden delimitarse las configuraciones de los espacios urbanos y sociales que habitan los jóvenes y que los habitan.

\section{Desde el espacio público}

En este entorno los sujetos jóvenes pasan a segundo plano. La obra paradigmática de esta perspectiva se encuentra en el texto coordinado por Jaume Trilla, Jóvenes y espacio público (2011), cuyos capítulos reflejan principalmente la situación de Barcelona, incluyendo también otros casos como los de la Ciudad de México, Medellín o Turín. El texto es clave, en especial porque cuenta con la colaboración de dos reconocidos investigadores sobre la ciudad como son Manuel Delgado y Jordi Borja, quienes parten de su expertisse sobre el análisis del espacio urbano, para posteriormente acercarse de forma colateral a la presencia de un solo tipo de jóvenes visibles ya sea por su estética o por su capacidad organizativa con fines de activismo político, 
dejando fuera del foco de atención a otras juventudes diversas entre sí por género, edad, clase, raza, etnia, estilo, nivel organizativo, etcétera.

En esta vía, los jóvenes son concebidos como una ventana para observar cómo se construye el espacio público, pero quizá también cómo se reflejan en lo juvenil las tensiones que se viven en él. En este punto habría que pensar en las distintas definiciones que existen de espacio público como lugar de interés ya sea colectivo, de encuentro, de posibilidad, de organización, de reconocimiento de las diferencias, o de homogeneidad, de miedo, de inseguridad, de anonimato, de conflicto, de exclusión, etcétera. Ante estas posibilidades, coincidimos con Maritza Urteaga (2013) cuando propone que existen diversos estilos de uso del espacio público por parte de la población en general, y en particular de los jóvenes según su género, edad, estrato socioeconómico y etnia.

\section{Hacia una propuesta teórico-metodológica}

En el acápite anterior planteamos dos posibilidades para analizar la relación de los jóvenes con el espacio público, ya sea desde una concepción lineal y unilateral en la que se aborda la presencia de los jóvenes en el espacio público, o desde la atención primordial en el espacio público, para mostrar en segundo plano las prácticas que llevan a cabo los jóvenes en él.

La propuesta que aquí se presenta es que se trata de una relación compleja y dinámica que deviene de distintas intersecciones en las relaciones de poder, lo cual da como resultado configuraciones distintas de ser y estar joven en el espacio público, algunas de ellas como reproductoras del "deber ser", otras más como formas disidentes de ese "deber ser" — desde una posición creativa y creadora, o destructiva y autodestructivao, las menos, en demanda de transformaciones sustanciales que permitan su presencia y participación activa, incluyente y democrática.

Consideramos que, para abordar lo que hemos denominado como la dupla jóvenes-espacio público, es necesario revisar desde qué posicionamiento se está partiendo en la concepción de lo juvenil y qué lugar toma el sujeto en relación con el espacio, sin perder de vista la influencia que ejerce el espacio público urbano en la conformación de la experiencia juvenil, lo cual por falta de espacio en este texto quedará pendiente para desarrollar en el futuro. Por el momento, se propone que, para estudiar la vinculación de los jóvenes con el espacio público, es importante colocar la experiencia juvenil en el centro del análisis y descolocarse de los dualismos, binarismos y dicotomías, es decir, pensar en la dupla jóvenes-espacio público como un entramado dinámico de conceptos, categorías, características, relaciones sociales de poder, contextos, escenarios, dimensiones y experiencias.

En la bibliografía consultada distintas formas de dar cuenta de estos entramados, aunque también es fácil verificar que en la mayoría de esas investigaciones se pasan por alto, por lo que los resultados y las reflexiones que se desprenden de ellas resultan insuficientes para comprender la complejidad de la dupla señalada. Por lo mismo, es pertinente, desde nuestra opinión, advertir sobre una serie de procedimientos metodológicos que podrían orientar el trabajo investigativo en las diversas temáticas que se han venido abordando sobre jóvenes y espacio público, especialmente en las últimas dos décadas, con miras a fortalecer el conocimiento generado hasta ahora.

\section{Caracterizar a los jóvenes y el o los espacios estudiados en su diversidad}

Si bien en los estudios recientes sobre juventudes puede verse una especificación más acotada de las características del sujeto juvenil en el que centramos la atención, aún se tiende a generalizar la experiencia de los jóvenes, dando por hecho características específicas de los sujetos de investigación como son la misma edad, el género, la orientación sexual, la etnicidad, la raza, la clase social y el territorio. Otro punto que puede observarse es la focalización en lo grupal, y muy poco en la experiencia individual de las y los jóvenes, ello quizá motivado por la representación recurrente de que las personas que se suelen ubicar en esta categoría social marcan una tendencia muy particular por agruparse. 
No obstante, consideramos que debe ponerse más atención en el individuo joven, y no sólo en las formas de grupalidad juvenil.

En suma, de manera recurrente se obvian las diferencias que implica el ser hombre o mujer, heterosexual o de la diversidad sexual, menor o mayor de edad, mestizo, indígena, extranjero, católico, cristiano, afrodescendiente, empobrecido, privilegiado, rural, urbano o migrante, sin detenerse en profundidad en la manera en que dichas condiciones moldean la propia experiencia juvenil; por tanto, es un punto de partida caracterizar cada una de estas particularidades en relación con el sujeto juvenil de estudio y ponerlas en juego dentro del análisis, no para clasificar o estereotipar a las personas jóvenes, sino para mostrar diferencias, especificidades y similitudes que en todo caso pueden ayudar a pensar en el tipo de políticas públicas que se requieren o son pertinentes. Además, dicha caracterización ayudará a mostrar las relaciones sociales de poder en las que se ubica el sujeto de investigación, así como sus posibilidades de acción, de expresión y de participación en los diferentes ámbitos sociales.

Junto con la caracterización del sujeto de investigación está la delimitación conceptual del espacio, en este caso del espacio público, tomando en cuenta las valoraciones, los simbolismos y la relación que presenta con el espacio privado, doméstico y privatizado, cada uno de ellos caracterizados y pensados como un continuum que permite observar sus interrelaciones, paradojas y simultaneidades, ayudando a romper con las miradas dicotómicas y jerarquizantes que se han instalado para vivirlos y estudiarlos.

A propósito de estas dimensiones, reflexionar y analizar las relaciones que se establecen en el espacio público se torna de gran importancia para ubicar las diferencias, desigualdades y asimetrías, pero también lo común, lo colectivo y las posibilidades de reconocimiento, de encuentro y de organización que ayudarán a comprender de mejor manera la dupla señalada.

Asimismo, se han realizado importantes esfuerzos por parte de los investigadores para mostrar la diversidad y heterogeneidad de experiencias de lo juvenil en relación con el espacio público a partir de distintas dimensiones, con el fin de cuestionar la concepción hegemónica y de sentido común que a la fecha prevalece con respecto a los jóvenes en México: varón, urbano, mestizo, pobre y transgresor.

En este sentido, diversos autores han visibilizado la presencia y experiencia de los jóvenes indígenas en el espacio público, como Urteaga (2007), García Álvarez (2012), Meneses (2016a), López (2017) y Meneses y López (2017), entre otros. No obstante, consideramos que en el caso mexicano la dimensión racial no ha sido desarrollada, a diferencia de los trabajos provenientes de España y de Estados Unidos, naciones a las que se les impuso la necesidad de problematizar esta matriz debido al creciente flujo migratorio de personas provenientes de otros países. Esto quizás se debe a que hasta hace poco se concebía que México era solamente país de tránsito de migrantes hacia Estados Unidos, cuestión que definitivamente está cambiando debido a la reconfiguración geopolítica mundial y del país vecino del norte que ha obligado a miles de centroamericanos, y más recientemente haitianos, a asentarse en nuestro país, sin contar las migraciones provenientes de otros países.

Con respecto a la clase social, resulta abrumadora la cantidad de trabajos que se han dedicado a analizar la experiencia de los jóvenes pobres, excluidos y marginados del espacio público, frente al escasísimo conocimiento que aún se tiene sobre los jóvenes de élite. Empero, es importante mencionar los trabajos de Urteaga (2013), Saraví (2015) y Rodríguez, Solares y Zabala (2009), como unos de los pocos estudios que analizan la experiencia urbana de los jóvenes de clases altas.

El análisis de la vinculación de los jóvenes y el espacio público con una perspectiva de género es imprescindible; no obstante, suele constituir una de las reflexiones más obviadas, pues se piensa erróneamente que género es igual a mujeres, olvidando que es un concepto que hace referencia a una relación social primaria de poder (Scott, 1996), la cual marca una diferencia tanto para los hombres, como para las mujeres y otros sujetos de género en la experiencia social, en este caso en la experiencia de lo juvenil y de 
la relación que se establece con el espacio público. En esta línea ha predominado un sesgo analítico que ni siquiera se reconoce, pues definitivamente se deja de lado la impronta que el género tiene en la vida de los varones jóvenes y en la construcción de su experiencia juvenil, rubricada en los más importantes estudios sobre las juventudes por la experiencia masculina.

Ahora bien, en el caso de que se haga referencia a la experiencia de las mujeres jóvenes, generalmente se efectúa desde el ámbito del riesgo y la violencia, sobre todo de tipo sexual, que pueden sufrir en el espacio público, la mayor parte del tiempo sin contar con datos y referencias sobre la manera en que las mujeres y los sujetos de la diversidad sexual resisten ante estas violencias (Rodó de Zárate, 2015) y, menos aún, de las vivencias e itinerarios positivos que estos sujetos experimentan (Del Valle, 2010), temas que consideramos necesario abordar en lo inmediato con la finalidad de ser incluyentes desde el propio planteamiento de los problemas de investigación acerca de experiencias relacionadas con la construcción social del género y de la edad (López, 2016) que se despliegan y se constituyen en el espacio público. Por tanto, una tarea a considerar en esta propuesta metodológica es mantener abierta la mirada a la diferencia entre los sexos y a las identidades genéricas, así como a las diferencias de edad que se observan entre los propios jóvenes, aspecto poco referenciado en la literatura.

Observamos que, aun cuando se repite una y otra vez que la juventud es una construcción social relacionada arbitrariamente con una edad relativa, lo cierto es que se sigue usando de manera omniabarcativa de una diversidad de experiencias diferenciadas, pues no es lo mismo ser menor de edad que mayor de edad y, por obvio que resulte, no es lo mismo tener 15 años, que 29. Las experiencias, las expectativas y las necesidades son inevitablemente distintas, razón por la cual resulta necesario segmentar y especificar claramente los rangos de edad de los sujetos de nuestras observaciones.

Más aún, las condiciones de vida cada vez más inciertas y flexibles para las mayorías han llevado a prolongar esta etapa etaria, lo que ha dado como resultado una moratoria social extrema que lleva a considerar joven a todo aquel que posea ciertos rasgos, estilos o características tales como empleos informales o desempleo, la cohabitación en casa de los padres hasta edades prolongadas, o la identificación con diversas grupalidades juveniles, lo cual conlleva una peligrosa prolongación de lo que se considera ser joven tras la que se oculta la irresponsabilidad del Estado de garantizar el acceso al pleno ejercicio de los derechos a la educación, la salud, el empleo o la vivienda.

Finalmente, en cuanto al territorio, éste se ha concebido principalmente desde la posición dicotómica de lo rural/urbano y del desarrollo/ subdesarrollo, dejando fuera de la reflexión, sobre todo en el campo de los estudios sobre juventud, el entramado territorial del cual se parte para el análisis. Esto obliga a diferenciar el tipo de espacio público y el tipo de relaciones que se tejen al respecto, ya sea en las periferias o en los centros tanto de las grandes ciudades, como de las ciudades medias, de los municipios y de las comunidades, tema que desarrollaremos a profundidad en un siguiente artículo.

\section{Contextualizar la vinculación en el tiempo y en los diferentes escenarios geopolíticos}

Este procedimiento implica historizar la vinculación de los jóvenes con el espacio público en términos de representaciones e imaginarios sociales, así como de usos, presencias y prácticas, pero también de las políticas públicas instaladas en lo próximo, lo local, lo regional, lo nacional y lo global. Son estos algunos escenarios de lo espacial de los que no siempre estamos dando cuenta y, menos, de la interrelación que guardan con las diversas experiencias juveniles.

Además, debe profundizarse de manera rigurosa y responsable en los contextos de violencia que, como en el caso mexicano, redefinen, y en buena medida determinan, las posibilidades y restricciones para ser y vivir la juventud, a tal punto que José Manuel Valenzuela (2012 y 2015) propone el concepto de "juvenicidio" para explicar un aumento considerable de víctimas mortales en edades jóvenes, muchas de ellas producto de muertes violentas y homicidios dolosos. 
No obstante, conviene señalar que, desde nuestro punto de vista, no cualquier joven es "asesinable" por el solo hecho de tener una edad determinada, pues la edad de las personas está cruzada por otras condiciones sociales tales como el género, la clase social o la pertenencia étnica, dimensiones que, en conjunto, vuelven "más asesinables" — retomando el término que utiliza el mismo autor - a jóvenes varones, pobres, etnizados y racializados, en comparación con otras personas jóvenes.

\section{Analizar cómo operan las diferenciaciones sociales}

Esto implica ir un paso más allá de la caracterización del sujeto de investigación, pues tiene que ver con un trabajo intersubjetivo y reflexivo entre el sujeto cognoscente y el sujeto cognoscible con los objetivos de plantear problemáticas y condiciones para la investigación y de mantener una autovigilancia epistemológica en el proceso investigativo y en los datos generados en el mismo. Esto permitirá regresar constantemente a la óptica del propio investigador, al lugar y a las condiciones desde las cuales está observando y desde donde produce conocimiento, elementos que sin duda impactarán en el tipo de resultados que arroje la investigación realizada. Además, la caracterización de los sujetos en razón de edad, género, sexualidad, etnia, raza, clase y territorio ayudará a pensar en el predominio o no de la perspectiva adulta en la construcción del espacio público, pero también en su generización, etnización, racialización, gentrificación, degradación, segregación e inclusión/exclusión.

Identificar a los distintos actores que intervienen en la relación jóvenes-espacio público

Por un lado, es central referirse en este procedimiento al papel que desempeñan el Estado, los gobiernos en sus distintos niveles, la Iglesia, el mercado, los organismos internacionales y las organizaciones no gubernamentales y de la sociedad civil, así como el ejercicio de los derechos, en las configuraciones concretas que adquiere la dupla jóvenes y espacio público.
Por otro lado, consideramos necesario incluir en el análisis del orden, así como de las violencias y los conflictos, el papel de las normas que regulan dicha relación y los modos de cuestionar, transgredir y transformar, o, en su caso, acatar dicha normatividad.

\section{Definir la posición o posiciones de los jóvenes en el espacio público}

Por último, creemos que, para ofrecer un conocimiento amplio y profundo de la relación entre jóvenes y espacio público, no hay que dejar de definir desde qué posición o posiciones se piensa a los jóvenes dentro de dicho espacio. En general, se parte de perspectivas positivas y optimistas o, por el contrario, negativas y pesimistas. Sin embargo, en lo empírico más bien se observan posicionamientos de los jóvenes en el espacio público con diferentes tesituras que mezclan ocupación, apropiación, disputa, evasión y participación, que se conjugan con expresiones, prácticas y acciones que dotan a los jóvenes de un papel relevante en la construcción del espacio público, aunque también en su extinción, reducción y destrucción.

De acuerdo con lo expuesto en los procedimientos arriba enunciados, puede concluirse que la dupla jóvenes-espacio público implica una relación dinámica, impermanente y conflictiva, que a su vez expresa una multiplicidad de experiencias determinadas en buena medida por la misma edad, el género, la clase, la raza, la etnia y el territorio, todo esto colocado en relaciones de poder específicas, de suerte que los marcos metodológicos para el abordaje y el análisis de tal dinamicidad también deben ser replanteados y puestos en juego en el campo de investigación.

Es importante mencionar que, desde nuestra experiencia investigativa, nos hemos inclinado hacia una perspectiva cualitativa, por lo que apelamos a la riqueza del método etnográfico con todas sus técnicas de recolección de datos, tales como entrevistas, observación participante, diarios de campo, itinerarios espaciales, fotografías, videos, mapas y cartografías simbólicas, exploraciones territoriales participativas, etcétera, pero sin descartar la posibilidad de incluir 
métodos y técnicas cuantitativas para enriquecer la información y los análisis de ésta, en la línea que aquí se ha planteado. Mostrar datos duros sigue siendo una estrategia de convencimiento para que los tomadores de decisiones dirijan la vista hacia las problemáticas que tienen las y los jóvenes en el espacio público. En esta misma tesitura, es importante incorporar la perspectiva y el papel de los medios de comunicación en la conformación de tal dupla, en la que los propios jóvenes imponen la necesidad de aproximarse a ellos de nuevas formas y, en este sentido, el uso de la tecnología, Internet y las redes sociales con fines de construcción de datos es ineludible.

Además, como un esfuerzo por tratar de desmontar la perspectiva adultocéntrica de los estudios sobre jóvenes y espacio público, ha de proponerse también el diseño de una nueva metodología que haga partícipes a los propios jóvenes sujetos de nuestras observaciones desde el mismo diseño, aplicación y análisis de los resultados; esta propuesta puede realizarse a partir de metodologías dialógicas, participativas o colaborativas, o de otras que tengan como finalidad llevar a cabo procesos investigativos más horizontales, en los que se considere a los jóvenes como conocedores principales de sus vidas y experiencias, las cuales son interpretadas por los sujetos jóvenes de acuerdo con la condición, situación y posición social que tienen en el entramado social. Por tanto, debe considerarse una ética para investigar que sea altamente respetuosa de las visiones, opiniones y perspectivas que aportan las personas jóvenes en los procesos investigativos, y de las cuales hacemos usos no siempre transparentes en la academia y en la política pública.

Hasta aquí hemos propuesto una serie de pasos que consideramos que es indispensable tener en cuenta y poner en práctica ante el interés por abordar la dupla jóvenes-espacio público. Pensamos que, para comenzar la discusión y la propuesta de una metodología que aborde el segundo de los elementos de la dupla, es relevante inclinarse hacia una concepción del espacio público que considere la interdisciplinariedad para su definición, entre otras perspectivas, haciendo eco de:
- Las propias concepciones del espacio público, la calle, la esquina, que desde el estudio de las juventudes se han propuesto, principalmente en su vertiente socioantropológica.

- Los aportes de la geografía feminista para analizar la relación intrínseca entre espacio privado, espacio doméstico y espacio público, así como entre género, cuerpo y espacio.

- La especificidad de la noción de espacio público frente a otros términos, tales como espacio, espacialidad, territorio, territorialidad o lugar, cuya discusión surge de la geografía humana.

- Los debates que, desde la ciencia política, la sociología y la antropología, se vierten sobre la relación que se establece entre identidad, poder, exclusión/inclusión, segregación, desigualdad y espacialidad.

\section{Reflexiones finales}

En estas páginas hemos efectuado una primera aproximación a la literatura publicada en las últimas dos décadas sobre jóvenes y espacio público, agrupando los diferentes trabajos revisados en por lo menos cuatro temáticas recurrentes que, sin duda, invitan a pensar en la posible consolidación de una línea de investigación que une el estudio de las juventudes con el espacio público.

Sin embargo, una problemática detectada en la literatura revisada hasta el momento y que podría frenar la consolidación de tal línea de indagación es que, desde nuestra experiencia y opinión, se observa una falta de procedimientos claros y delimitados para abordar la dupla jóvenes y espacio público, por lo que queda la sensación de que hay muchas más generalizaciones que concreciones, hallazgos sesgados o incompletos, algunas veces desfasados, que no permiten mostrar la densidad que se construye tras esta temática. Se encontraron además textos cargados de juicios de valor que devienen de una serie de premisas, prenociones y prejuicios que se revelan en las definiciones o falta de ellas en cuanto a lo juvenil y al espacio público, así como dificultades para mostrar su diversidad, sus múltiples dimensiones espaciales y temporales, el papel del 
Estado, del mercado, de las normas, de los conflictos y de las violencias de las que no se puede dejar de dar cuenta.

Por todo ello, consideramos relevante pensar en una serie de procedimientos metodológicos que puedan contribuir a subsanar las debilidades señaladas y que de alguna manera se retoman de las mismas lecturas y de nuestra experiencia investigativa, para conformar las premisas de un modelo de análisis que se pretende coherente, flexible y en continua construcción de acuerdo con la propia historia social y cultural y con los intereses investigativos, pero sobre todo que tome en cuenta las preocupaciones de los propios sujetos de investigación, las y los jóvenes, quienes cobran en esta propuesta metodológica un lugar protagónico y activo.

\section{Notas}

${ }^{1}$ Se trata del Seminario Jóvenes, Espacio Público y Ciudadanía,llevado a caboen febrero de 2016 en el Centro de Investigaciones Interdisciplinarias en Ciencias y Humanidades, y del Segundo Seminario Internacional Jóvenes y Espacio Público, realizado en febrero de 2017 en el Instituto de Investigaciones Sociales, ambos en la Universidad Nacional Autónoma de México.

2 Por economía del lenguaje se utiliza el artículo masculino, pero nos referimos siempre a los diversos sujetos de género y de la experiencia juvenil. No obstante, hay momentos en que es preciso nombrar a las mujeres o a los varones jóvenes para enfatizar su presencia y experiencia.

${ }^{3}$ Centrada en la perspectiva adulta y normativa que se impone a los jóvenes de forma autoritaria y hegemónica.

${ }^{4}$ Objetivo de la mayoría de los programas de gobierno que apelan a la recuperación del espacio público con fines de prevención del delito, por ejemplo.

${ }^{5}$ Que en el caso mexicano se expresan en una serie de instrumentos jurídicos que penalizan hasta con cárcel a los escritores de grafiti, tal como la creación en 2004 de la Unidad Antigraffiti en la Ciudad de México, hasta llegar a casos de asesinato de grafiteros a manos de la policía, como fue el caso de Ricardo Cadena, asesinado por un policía por pintar una pared en las calles de Cholula, Puebla, en 2015.
${ }^{6}$ Se trata de un concepto en construcción, aún debatible, particularmente cuando se pretende análogo al de feminicidio, el cual alude al acto de violencia extrema en el que las mujeres son asesinadas por el solo hecho de ser mujeres, independientemente de otras condiciones sociales; por otra parte, el incremento de asesinatos de personas jóvenes está cruzado por otros ordenadores sociales que, si bien atraviesan el género, éste no determina "la muerte artera" de los jóvenes a quienes se refiere Valenzuela.

\section{Referencias}

Arditi, Benjamin (2015). "La política distribuida de los rebeldes del presente: la acción en la era de la Web 2.0". Documento de Trabajo No. 4 del Proyecto PAPIIT 308313 "Política viral y redes: invención y experimentación desde el Magreb al \#Yosoyl32". México: Dirección General de Asuntos del Personal Académico de la UNAM.

Batallán, Graciela, Silvana Campanini, Elías Prudant, Enrique Lara y Soledad Castro (2009). "La participación política de jóvenes adolescentes en el contexto urbano argentino. Puntos para el debate". En Última Década, 30: 41-66.

Berroeta, Héctor y Juan Sandoval (2014). "Protestas, participación y educación pública: discursos sobre lo público en las movilizaciones estudiantiles en Chile". En Educar em Revista, 53: 19-38.

Bohórquez-Pereira, Giovanni, Blanca Aracely López Rueda y Astrid Suárez González (2016). “Jóvenes y sus relaciones con los espacios urbanos en una ciudad capital". En Ánfora, 23(40): 75-98.

Castro Luque, Ana Lucía y Blanca Zepeda Bracamonte (2004). "El café Internet: un espacio real para la virtualidad. Jóvenes y ciberespacio en el noroeste de México". En Región y Sociedad, 31: 163-208.

Chaves, Mariana (2010). Jóvenes, territorios y complicidades. Una antropología de la juventud urbana. Buenos Aires: Espacio Editorial.

Del Valle, Teresa (2010). "El derecho a la movilidad libre y segura”. En Virginia Maquieira D’Angelo (coord.), Mujeres, globalización y derechos humanos. Madrid: Cátedra. 
Eseverri Mayer, Cecilia (2015). “Jóvenes sin vínculos. El papel de las estructuras intermedias en un espacio urbano desfavorecido". En Reis. Revista Española de Investigaciones Sociológicas, 150: 23-39.

Espinosa Spínola, María (2009). “La 'chavos banda’ como forma de organización alternativa a la familia entre los menores en situación de calle en la Ciudad de México". En Gazeta de Antropología, 25(1).

Espinosa Spínola, María (2012). "Discursos, narrativas y percepciones entre los y las niñas de la calle en torno a su huida". En Desacatos. Revista de Antropología Social, 40: 97-110.

García Álvarez, Luis Fernando (2012). Jóvenes indígenas en contextos metropolitanos. La construcción de lo juvenil en una comunidad mixteca en el Área Metropolitana de Monterrey, Nuevo León. Tesis de Maestría en Antropología Social, Escuela Nacional de Antropología e Historia, México.

Hernández Cabrera, P.M. (2001). "La construcción de la identidad gay en un grupo gay de jóvenes de la Ciudad de México. Algunos ejes de análisis para el estudio etnográfico". En Desacatos. Revista de Antropología Social, 6: 63-96.

Hine, Christine (2000). Etnografía virtual. Barcelona: Editorial UOC.

Jasso, Carmina (2015). "El desorden y la incivilidad en el espacio público. Aproximaciones de política pública para su planteamiento". En Revista Alter. Enfoques Críticos, VI(12): 51-67.

Lopera Molano, Á.M. y P. Coba Gutiérrez (2001). "Intervención del espacio público: percepción ciudadana del grafiti en la ciudad de Ibagué, Colombia". En Revista Encuentros, 14(1): 55-71.

López Guerrero Jahel (2016). “Aportes de los estudios feministas al análisis de la interrelación entre género y edad: claves para abordar la experiencia juvenil de las mujeres". En Norma Blázquez Graf y Martha Patricia Castañeda Salgado (coords.), Lecturas críticasen investigación feminista. México: CEIICH-UNAM.

López Guerrero, Jahel (2017). Mujeres jóvenes indígenas migrantes en la Zona Metropolitana del Valle de México: Condiciones estructurales y subjetividades en la construcción de su experiencia juvenil. México: CEIICH-UNAM.
Makowski, Sara (2007). "Ciudad de México: territorios de la exclusión”. En Espaço Plural, VIII(17): 9-16.

Meneses Reyes, Marcela e Itandehui Franco (2018). "De la transgresión a la institucionalización. La gráfica política en la reconfiguración del espacio público en la ciudad de Oaxaca". En Patricia Ramírez Kuri (coord.), La erosión del espacio público en la ciudad neoliberal. México: IIS-UNAM.

Meneses Reyes, Marcela y Jahel López Guerrero (2017). "Jóvenes indígenas migrantes: apropiación del espacio público en la Ciudad de México". En Assis da Costa Oliveira y Lúcia Helena Vitalli Rangel (coords.), Juventudes indigenas: estudos interdisciplinares, saberes interculturais - conexões entre Brasile México. Brasil: E-Papers.

Meneses Reyes, Marcela (2008). "Juventud, espacio urbano y exclusión social”. En Rolando Cordera, Alicia Ziccardi y Patricia Ramírez Kuri (coords), Pobreza, desigualdad y exclusión social en la ciudad del siglo XX. México: IIS-UNAM, Siglo XXI Editores.

Meneses Reyes, Marcela (2016a). "Jóvenes indígenas migrantes en la Alameda Central. Disputas pacíficas por el espacio público". En Iztapalapa. Revista de Ciencias Sociales y Humanidades, 80(37): 39-68.

Meneses Reyes, Marcela (2016b). "Ni derecho al centro tenemos'. Jóvenes artistas gráficos en el espacio público de Oaxaca, 2006". En Espacialidades, 6(1): 144-166.

Morfín, Catalina (2011). "Jóvenes en acciones colectivas y movimientos sociales para redefinir los espacios públicos y las prácticas ciudadanas". En Revista Latinoamericana de Ciencias Sociales, Niñez y Juventud, 9(1): 61-79.

Omaña Aldana, Luis Omar (2011). "La socialización del espacio público, una alternativa para la adolescencia en la periferia urbana". En Fermentum. Revista Venezolana de Sociología y Antropología, 21(61): 159-175.

Ortega Nuere, Cristina, Idurre Lazcano Quintana y Rocha Teixeira Baptista (2015). "Espacios de ocio para jóvenes, de la monitorización a la autogestión”. En Pedagogía Social. Revista Interuniversitaria, 25: 69-89.

Pérez, Miguel, Rodrigo Salcedo y Gonzalo Cáceres (2012). "Apropiación y control social en un centro comercial de Santiago: prácticas socioespaciales y significaciones adolescentes". En EURE, 38(113): 53-75. 
Pérez López, Ruth y Lucía Barragán Rodríguez (2012). "Construcción social de un espacio público en la Ciudad de México: la Plaza Zarco y sus jóvenes". En Nueva Antropología, XXV(76): 13-32.

Portillo, Maricela (2014). "Mediaciones tecnocomunicativas, movilizaciones glogales y disputas por la visibilidad en el espacio público. Análisis del surgimiento del \#YoSoyl32”. En Argumentos, 27(75): 173-190.

Ravelo Blancas, Patricia y Héctor Domínguez Rubalcaba (2010). "Ciudad Juárez: asedios a la ciudadanía y cancelación de la vida urbana". En El Cotidiano, 164: 5-10.

Reguillo, Rossana (2012a). "Navegaciones errantes. De músicas, jóvenes y redes: de Facebook a Youtube y viceversa". En Comunicación y Sociedad, 18: 135-171.

Reguillo, Rossana (2012b). "De las violencias: caligrafía y gramática del horror”. En Desacatos, 40: 33-46.

Reguillo, Rossana (2012c), "Reflexiones iniciales en torno a \#YoSoyl32”. En Revista Magis Iteso, 28 de mayo. Disponible en: https://bit.ly/2GrDngY.

Rodó de Zárate, Maria (2015) “El acceso de la juventud al espacio público en Manresa. Una aproximación desde las geografías feministas de la interseccionalidad". En Scripta Nova. Revista Electrónica de Geografía y Ciencias Sociales, XIX(504): 1-26.

Rodríguez Ostria, Gustavo, Humberto Solares Serrano y María Lourdes Zabala Canedo (2009). "Jóvenes, miedo y espacio urbano en Cochabamba". En Tinkazos. Revista Boliviana de Ciencias Sociales, 25.

Rovira Sancho, Guiomar (2013a). "La Primavera Mexicana \#YOSOY132, la comunicación y el proceso electoral de 2012 en México". En Fundación Betiko. Disponible en: http://fundacionbetiko.org/ wp-content/uploads/2013/03/Articulo-Yosoyl32Guiomar.pdf.

Rovira Sancho, Guiomar (2013b). “Activismo mediático y criminalización de la protesta: medios y movimientos sociales en México". En Convergencia. Revista de Ciencias Sociales, 20(61): 35-60.

Rovira Sancho, Guiomar (2014). "El \#YoSoyl32 mexicano: la aparición (inesperada) de una red activista”. En Revista CIDOB d'Afers Internacionals, 105: 47-66.
Saraví, Gonzalo (2004). "Segregación urbana y espacio público: los jóvenes en enclaves de pobreza estructural". En Revista de la CEPAL, 83: 33-48.

Saraví, Gonzalo(2015). Juventudes fragmentadas. Socialización, clase y cultura en la construcción de la desigualdad. México: FLACSO, CIESAS.

Scott, Joan W. (1996). "El género: una categoría útil para el análisis histórico”. En Marta Lamas (comp.), El género: la construcción cultural de la diferencia sexual. México: PUEG-UNAM.

Trilla, Jaume (coord.) (2011). Jóvenes y espacio público: del estigma a la indignación. Barcelona: Edicions Bellaterra.

Tully, Claus y Claudio Alfaraz (2012). "Jóvenes, espacio y tecnología. La configuración de las relaciones sociales en la vida cotidiana". En Propuesta Educativa, 38: 59-68.

Urteaga, Maritza (2007). "Jóvenes e indios en la ciudad". En Tramas, 28: 85-120.

Urteaga, Maritza (2013). "Espacios públicos de los jóvenes y culturas juveniles". En José Luis Calva (coord.), Los jóvenes de hoy: presente y futuro. México: Juan Pablos Editor.

Urzúa Martínez, Sergio (2015). “¿Cómo marchan los jóvenes en el Chile de postdictadura? Algunas notas acerca de la apropiación del espacio público y el uso político del cuerpo". En Última Década, 42: 39-64.

Valenzuela, José Manuel (2012). Sed de mal. Feminicidio, jóvenesyexclusión social. México: El Colegio de la Frontera Norte, Universidad Autónoma de Nuevo León.

Valenzuela, José Manuel (coord.) (2015). Juvenicidio. Ayotzinapa y las vidas precarias en América Latina y España, Barcelona: NED Ediciones, Guadalajara: ITESO, Tijuana: COLEF.

Víquez Abarca, Rosibel y Mauricio Leandro Rojas (2006) "Espacios públicos y percepción de los adolescentes urbanos". En Reflexiones, 85: 117-129.

Vommaro, Pablo (2014). "La disputa por lo público en América Latina. Las juventudes en las protestas y en la construcción de lo común". En Nueva Sociedad, 251: 55-69.

Zenil Medellín, Mónica Eugenia (2010). Construcción ciudadana y apertura de espacios públicos. Prácticas sociales de jóvenes en la Ciudad de México. Tesis para obtener el grado de doctora en Ciencias Políticas y Sociales con orientación en Sociología. México: UNAM. 